3-1-2008

\title{
Dipole in a Magnetic Field, Work, and Quantum Spin
}

Robert J. Deissler

Cleveland State University, r.deissler@csuohio.edu

Follow this and additional works at: https://engagedscholarship.csuohio.edu/sciphysics_facpub

Part of the Physics Commons

How does access to this work benefit you? Let us know!

Publisher's Statement

Copyright 2008 American Physical Society. Available on publisher's site at http://pre.aps.org/ abstract/PRE/v77/i3/e036609.

\section{Original Citation}

Deissler, Robert J. "Dipole in a Magnetic Field, Work, and Quantum Spin." Physical Review E 77 (2008): 36609.

\section{Repository Citation}

Deissler, Robert J., "Dipole in a Magnetic Field, Work, and Quantum Spin" (2008). Physics Faculty Publications. 72.

https://engagedscholarship.csuohio.edu/sciphysics_facpub/72

This Article is brought to you for free and open access by the Physics Department at EngagedScholarship@CSU. It has been accepted for inclusion in Physics Faculty Publications by an authorized administrator of

EngagedScholarship@CSU. For more information, please contact library.es@csuohio.edu. 


\title{
Dipole in a magnetic field, work, and quantum spin
}

\author{
Robert J. Deissler* \\ Physics Department, Cleveland State University, Cleveland, Ohio 44114, USA
}

(Received 28 February 2007; published 21 March 2008)

\begin{abstract}
The behavior of an atom in a nonuniform magnetic field is analyzed, as well as the motion of a classical magnetic dipole (a spinning charged ball) and a rotating charged ring. For the atom it is shown that, while the magnetic field does no work on the electron-orbital contribution to the magnetic moment (the source of translational kinetic energy being the internal energy of the atom), whether or not it does work on the electron-spin contribution to the magnetic moment depends on whether the electron has an intrinsic rotational kinetic energy associated with its spin. A rotational kinetic energy for the electron is shown to be consistent with the Dirac equation. If the electron does have a rotational kinetic energy, the acceleration of a silver atom in a Stern-Gerlach experiment or the emission of a photon from an electron spin flip can be explained without requiring the magnetic field to do work. For a constant magnetic field gradient along the $z$ axis, it is found that the classical objects oscillate in simple harmonic motion along the $z$ axis, the total kinetic energy - translational plus rotational-being a constant of the motion. For the charged ball, the change in rotational kinetic energy is associated only with a change in the precession frequency, the rotation rate about the figure axis remaining constant.
\end{abstract}

DOI: 10.1103/PhysRevE.77.036609

PACS number(s): 45.20.dg, 45.40.-f, 03.50.De, 03.65.-w

\section{INTRODUCTION}

Place an atom in a nonuniform static external magnetic field and, because of the interaction between the magnetic moment of the atom and the magnetic field gradient, the atom will accelerate. This, of course, is what occurs in the classic Stern-Gerlach experiment. An important and fundamental question is whether or not the magnetic field is doing work on the atom. A thorough review of both the research and educational literature reveals that this question has not been adequately addressed. The only papers which address this question in any detail appear to be a paper by Goldstein [1], which analyzes a classical dipole in a uniform magnetic field, and a paper by Coombes [2], which discusses a semiclassical model of a hydrogen atom (no spin) in a nonuniform magnetic field. Both these papers point out that classically, a static magnetic field does no work. For the classical dipole in a uniform magnetic field, this is accomplished by any increase in the kinetic energy of precession or nutation coming at the expense of rotational kinetic energy about the figure axis. For the semiclassical hydrogen atom in a nonuniform magnetic field, any increase in translational kinetic energy comes at the expense of the internal energy of the atom.

As is well known, the source of the magnetic moment for an atom is the orbital motion of the electrons in the atom and the spin of the electrons $[3,4]$. If the only source of the magnetic moment were the orbital motion of the electrons, then the magnetic field would, in fact, not be doing work, any increase in the translational kinetic energy of the atom being associated with a decrease in the internal energy of the atom, as noted above for a semiclassical model of hydrogen. In the present paper, this statement will be shown for an atom within a fully quantum mechanical framework (nonrelativistic): Any increase in the expectation value of the translational

*URL: http://deissler.us; deissler@zubetube.com kinetic energy of an atom will be associated with a corresponding decrease in the expectation value of the internal energy of the atom.

If the spin contribution to the magnetic moment is included, then the situation is not as clear. The spin Hamiltonian for an electron is $H_{s}=-\boldsymbol{\mu}_{\boldsymbol{s}} \cdot \boldsymbol{B}$, where $\boldsymbol{\mu}_{\boldsymbol{s}}$ is the intrinsic magnetic moment of the electron and $\boldsymbol{B}$ is the magnetic field $[3,4]$. Often this term is referred to as a potential energy, in analogy with the potential energy for an electric dipole in an electric field, where the electric field of course can do work. By potential energy is meant the usual potential energy $U$ from which the force is derived by $\boldsymbol{F}=-\boldsymbol{\nabla} U$. If $H_{s}$ were a potential energy, then any change in $H_{s}$ would correspond to work done by the magnetic field, $W=-\Delta H_{s}$. Therefore one would arrive at the unsatisfying conclusion that the magnetic field would do work on the spin contribution to the magnetic moment, even though it does no work on the orbital contribution to the magnetic moment.

If the magnetic field is to do no work on the spin contribution to the magnetic moment, then the electron would need to have an intrinsic rotational kinetic energy associated with its spin. Since electron spin is inherent in the Dirac equation $[5,6]$, giving rise to an accurate value for the magnetic moment of the electron, any discussion of electron spin should consider the Dirac equation. As shown in the present paper, the notion of an intrinsic rotational kinetic energy for the electron is, in fact, consistent with the Dirac equation. This rotational kinetic energy would then be the source of energy for any increase in the translational kinetic energy of the atom related to the interaction of the spin magnetic moment with the magnetic field gradient, such as occurs in a SternGerlach experiment. It also explains the emission of a photon from an electron spin flip without requiring the magnetic field to do work.

In addition, if a static magnetic field could do work on a magnetic moment resulting from quantum spin, this would contradict what occurs classically. As noted previously, classically a static magnetic field cannot do work. It can only 
redirect motion. This is so since the magnetic force is always perpendicular to the velocity of the particle. Note that this will be the case even if there are constraints on the system, such as those occurring for a rigid rotator, as analyzed by Goldstein for a uniform magnetic field [1]. The fact that classically the magnetic field cannot do work will be discussed further in Sec. III.

Since no one appears to have previously analyzed the motion of a classical dipole in a nonuniform magnetic field and since much insight can be gained from such a study, two classical systems are studied: (i) a rotating charged ring in a nonuniform magnetic field and (ii) a spinning charged ball in a nonuniform magnetic field. Both these systems exhibit interesting behavior. For the rotating ring it is found that, if the magnetic field and a constant gradient in this field are directed along the $z$ axis and if the axis of rotation of the ring is along this axis, the ring undergoes simple harmonic motion along this axis, the kinetic energy being transferred alternately between translational and rotational motion. For the same magnetic field, even though there is a nonzero angle between the magnetic field vector and the rotation axis, the charged ball also oscillates in simple harmonic motion along the $z$ axis. Another interesting feature for the spinning charged ball is that, while the charged ball is oscillating along the $z$ axis, it is only the precession frequency that changes, the rotation rate about the figure axis remaining constant. Any change in the translational kinetic energy is thus compensated for by a change in the rotational kinetic energy associated with a change in the precession frequency. It is noted that this oscillatory translational motion is different from that of a point charge that is trapped in a nonuniform magnetic field (i.e., a "magnetic mirror") [7], where the magnetic field gradient has opposite signs at the two ends where the particle is reflected. For the cases of the rotating ring and the spinning charged ball, there is no change in the sign of the magnetic field gradient.

\section{A ROTATING CHARGED RING IN A NONUNIFORM MAGNETIC FIELD}

Consider a rotating charged nonconducting ring of charge $q$, mass $m$, and radius $a$ with the normal to the ring and the axis of rotation being along the $z$ axis and with a magnetic field and its gradient being along the positive $z$ axis. Since its divergence is zero, the magnetic field lines will converge toward the $z$ axis for increasing $z$, and therefore the magnetic field will have a component in the radial direction when off the $z$ axis. This radial component will create a force along the $z$ axis on the rotating ring given by

$$
F=\mu \frac{\partial B}{\partial z},
$$

where $B(z)$ is defined as the magnitude of the magnetic field vector on the $z$ axis. Using $\mu=i A / c$, where $i=q \omega / 2 \pi$ is the current generated by the charge rotating with angular frequency $\omega$, and $A=\pi a^{2}$ is the area of the ring, the equation for the velocity $v=d z / d t$ is [8]

$$
m \frac{d v}{d t}=\frac{q \omega a^{2}}{2 c} \frac{\partial B}{\partial z} .
$$

As a result of the ring's motion in the $z$ direction, the radial component of the magnetic field will also produce a torque on the ring. This torque, which is directed along the $z$ axis, may be found as follows. Because of the gradient in the magnetic field and the ring's motion in the $z$ direction, there will be a changing magnetic flux through the ring. According to Faraday's law of induction, the changing flux will produce an emf and an electric field along the ring given by

$$
\mathcal{E}=2 \pi a E=-\frac{1}{c} \frac{d}{d t}(B A)=-\frac{A}{c} \frac{\partial B}{\partial z} \frac{d z}{d t} .
$$

The electric field will produce a torque, $\tau=q E a$, on the ring. Combining this with Eq. (3), and noting that $\tau=m a^{2} d \omega / d t$, the equation for $\omega$ is

$$
m \frac{d \omega}{d t}=-\frac{q v}{2 c} \frac{\partial B}{\partial z} .
$$

From Eq. (2) and Eq. (4) it is easy to show that

$$
\frac{d}{d t}\left(\frac{1}{2} m v^{2}+\frac{1}{2} m a^{2} \omega^{2}\right)=0
$$

Therefore the total kinetic energy, translational plus rotational, is a constant of the motion and the magnetic field does no work. If the translational kinetic energy increases, the rotational kinetic energy must decrease and vice versa.

Eliminating $\omega$ from Eq. (2) and Eq. (4) gives

$$
\frac{d^{2} v}{d t^{2}}=-\left(\frac{q a \eta}{2 m c}\right)^{2} v,
$$

where $\eta=\partial B / \partial z$. For $\eta$ constant, this is an equation for simple harmonic motion. Therefore the ring will oscillate in the $z$ direction, kinetic energy being transferred alternately between translation and rotation. For $\eta$ constant, the angular frequency of oscillation for the simple harmonic motion is

$$
\omega_{s}=\frac{|q \eta| a}{2 m c} .
$$

Note that constant $\eta$ on the $z$ axis is easily satisfied by assuming that the $r, \theta$, and $z$ components of the magnetic field are

$$
B_{r}=-\frac{1}{2} \eta r, \quad B_{\theta}=0, \quad B_{z}=B_{0}+\eta z,
$$

which satisfy $\boldsymbol{\nabla} \cdot \boldsymbol{B}=0$ and $\boldsymbol{\nabla} \times \boldsymbol{B}=0$. For future reference, the components of the vector potential $\boldsymbol{A}$ (in the Coulomb gauge) from which $\boldsymbol{B}=\boldsymbol{\nabla} \times \boldsymbol{A}$ may be obtained are

$$
A_{r}=0, \quad A_{\theta}=\frac{1}{2} r\left(B_{0}+\eta z\right), \quad A_{z}=0 .
$$

Equation (2) and Eq. (4) may also be derived directly from the magnetic force equations by considering an infinitesimal section of the ring with charge $d q$. Because the ring in general is both rotating about the $z$ axis (with angular 
velocity $\omega=d \theta / d t$ ) and translating along the $z$ axis (with translational velocity $v=d z / d t$ ), there will be both a $\theta$ and a $z$ component of force on $d q$ given by

$$
d F_{\theta}=\frac{d q}{c} v B_{r} \quad \text { and } \quad d F_{z}=-\frac{d q}{c} a \omega B_{r} .
$$

Integrating over the ring then gives for the net torque $\tau$ and the net force $F_{z}$

$$
\tau=m a^{2} \frac{d \omega}{d t}=\frac{q}{c} a v B_{r} \quad \text { and } \quad F_{z}=m \frac{d v}{d t}=-\frac{q}{c} a \omega B_{r} .
$$

From Eq. (11) follows the conservation of kinetic energy, Eq. (5). If the magnetic field satisfies Eq. (8), then $B_{r}=$ $-(1 / 2) \eta r$ may be inserted into Eq. (11) giving Eq. (1), Eq. (2), and Eq. (4). Therefore the motion of the ring will be described by Eq. (2) and Eq. (4), regardless of the size of the ring. If the magnetic field does not satisfy Eq. (8), then Eq. (1), Eq. (2), and Eq. (4) still follow if the radius of the ring is assumed to be small.

Note that since Eq. (1) can be derived directly from the force equations as just shown, this is the correct expression for $F$ and not $F=\partial(\mu B) / \partial z$, since $\mu$ is not constant. Therefore $F$ cannot be written in terms of a potential energy. This again reinforces the point that we are not dealing with a potential energy associated with the interaction of the magnetic moment with the magnetic field.

The Lagrangian for this system is

$$
\mathcal{L}=\frac{1}{2} m v^{2}+\frac{1}{2} m a^{2} \omega^{2}+\frac{\omega a^{2} q}{2 c} B(z) .
$$

The last term in Eq. (12) is $\mu B$. It is easy to verify that Eq. (2) and Eq. (4) result from this Lagrangian. The generalized angular momentum [9] is

$$
L=\frac{\partial \mathcal{L}}{\partial \omega}=m a^{2} \omega+\frac{a^{2} q}{2 c} B(z) .
$$

Since $\mathcal{L}$ is independent of the angular coordinate $\theta$, or cyclic in $\theta, L$ is a constant of the motion. The Hamiltonian for this system is the kinetic energy. In terms of the momentum $p$ $=\partial \mathcal{L} / \partial v$ and the generalized angular momentum $L$, the Hamiltonian is

$$
H=\frac{p^{2}}{2 m}+\frac{1}{2 m a^{2}}\left(L-\frac{a^{2} q}{2 c} B(z)\right)^{2} .
$$

Equation (6) gives the equation of simple harmonic motion in terms of $v$. It would be desirable to have an equation in terms of $z$. The ring oscillates about the $z$ coordinate for which $\omega=0$. In terms of the generalized angular momentum, Eq. (13), this $z$ coordinate is given by

$$
B(z)=\frac{2 c}{a^{2} q} L .
$$

Assuming constant $\eta=\partial B / \partial z$, taking $B(z)=B_{0}+\eta z$, and using Eq. (2), gives for the equation of motion for $z$

$$
\ddot{z}=-\omega_{s}^{2}\left(z-z_{0}\right),
$$

where $\omega_{s}$ is given by Eq. (7) and

$$
z_{0}=\frac{2 c L}{q a^{2} \eta}-\frac{B_{0}}{\eta}
$$

the $z$ coordinate about which the ring oscillates. Since $L$ can take on any constant value, the ring can be made to oscillate about any $z_{0}$. Note that $z_{0}$ is also given by that $z$ which minimizes $\left[L-\left(a^{2} q / 2 c\right) B(z)\right]^{2}$ in Eq. (14).

\section{A SPINNING CHARGED BALL IN A NONUNIFORM MAGNETIC FIELD}

As pointed out in the Introduction, classically a static magnetic field cannot do work, since the magnetic force is always perpendicular to the velocity. This is true even for a macroscopic number of particles as the following argument shows. For $N$ localized charged particles, each of charge $q_{i}$, in a static external magnetic field, the magnetic field at the position of the $i$ th particle is

$$
\boldsymbol{B}_{i}=\boldsymbol{B}_{\text {ext }, i}+\boldsymbol{B}_{i n t, i},
$$

where $\boldsymbol{B}_{\text {ext }, i}$ is the external magnetic field and $\boldsymbol{B}_{\text {int,i }}$ is the magnetic field generated by the motion of the particles themselves. By external magnetic field is meant that the currents producing this field are far from and therefore unaffected by the $N$ charged particles or their motion. The work done per unit time on particle $i$ by the magnetic field is

$$
\frac{d W_{i}}{d t}=\frac{q_{i}}{c} \boldsymbol{v}_{i} \cdot\left(\boldsymbol{v}_{i} \times \boldsymbol{B}_{i}\right)=0 .
$$

From Eq. (18), the rate at which the magnetic field does work can be split into the rate at which the external magnetic field does work,

$$
\frac{d W_{e x t, i}}{d t}=\frac{q_{i}}{c} \boldsymbol{v}_{i} \cdot\left(\boldsymbol{v}_{i} \times \boldsymbol{B}_{e x t, i}\right)=0,
$$

and the rate at which the internal magnetic field does work,

$$
\frac{d W_{i n t, i}}{d t}=\frac{q_{i}}{c} \boldsymbol{v}_{i} \cdot\left(\boldsymbol{v}_{i} \times \boldsymbol{B}_{i n t, i}\right)=0 .
$$

Therefore the work done on the $i$ th particle by the static external magnetic field and the work done on the $i$ th particle by the internal magnetic field generated by the motion of the particles themselves are both zero. Note that the net work done by both these magnetic fields is also zero. If any work is done, it is done by electric fields produced by the charged particles or produced by changing magnetic fields associated with the motion of the charged particles [2]. Therefore even though $\boldsymbol{B}_{\text {int }, i}$ cannot do work directly, as shown by Eq. (21), it may do work indirectly, via the electric field generated by a changing $\boldsymbol{B}_{i n t, i}$. However, this is not the case for the static external field, $\boldsymbol{B}_{\text {ext }, i}$, since it is independent of time. The important point here is that classically a static external magnetic field cannot do work, whether directly, as shown by Eq. (20), or indirectly, since $\boldsymbol{B}_{\text {ext, } i}$ is static and therefore cannot generate an electric field. 
Now consider a spinning charged body with center of mass velocity $\boldsymbol{v}$ and angular velocity $\boldsymbol{\omega}$ in a magnetic field $\boldsymbol{B}$ given by a vector potential $\boldsymbol{A}$. The Lagrangian for this system is

$$
\begin{aligned}
\mathcal{L}= & \int\left[\frac{1}{2} \rho\left(\boldsymbol{r}^{\prime}\right)\left(\boldsymbol{v}+\boldsymbol{\omega} \times \boldsymbol{r}^{\prime}\right)^{2}\right. \\
& \left.+\frac{\rho_{q}\left(\boldsymbol{r}^{\prime}\right)}{c} \boldsymbol{A}(\widetilde{\boldsymbol{r}}) \cdot\left(\boldsymbol{v}+\boldsymbol{\omega} \times \boldsymbol{r}^{\prime}\right)\right] d V^{\prime},
\end{aligned}
$$

where $\rho$ is the mass density, $\rho_{q}$ is the charge density, $\widetilde{\boldsymbol{r}}=\boldsymbol{r}$ $+\boldsymbol{r}^{\prime}$ is the position vector in a fixed frame, $\boldsymbol{r}$ is the position vector of the center of mass, $\boldsymbol{r}^{\prime}$ is the position vector relative to the center of mass, and the integral is taken over the volume of the charge. The first term of this equation is simply the kinetic energy and the second term is the interaction term, describing the interaction between the charge and the magnetic field. Note that the magnetic field produced by the spinning charged body itself need not be considered, since the charged body is assumed to be rigid and therefore the internal forces have no effect on the ball's motion (radiative effects being neglected). If $\boldsymbol{v}=0$ and $\boldsymbol{B}$ is uniform, Eq. (22) reduces to the equation immediately preceding Eq. (13) in Goldstein [1], which considers a spinning charged body with no translational velocity in a uniform magnetic field. Equation (22) may be written as

$$
\mathcal{L}=\frac{1}{2} m v^{2}+\frac{1}{2} \boldsymbol{\omega} \cdot \boldsymbol{I} \cdot \boldsymbol{\omega}+\boldsymbol{R} \cdot \boldsymbol{v}+\boldsymbol{G} \cdot \boldsymbol{\omega},
$$

where $m$ is the total mass, $\boldsymbol{I}$ is the moment of inertia tensor,

$$
\boldsymbol{R}(\boldsymbol{r})=\frac{1}{c} \int \rho_{q}\left(\boldsymbol{r}^{\prime}\right) \boldsymbol{A}\left(\boldsymbol{r}+\boldsymbol{r}^{\prime}\right) d V^{\prime},
$$

and

$$
\boldsymbol{G}(\boldsymbol{r})=\frac{1}{c} \int \rho_{q}\left(\boldsymbol{r}^{\prime}\right)\left[\boldsymbol{r}^{\prime} \times \boldsymbol{A}\left(\boldsymbol{r}+\boldsymbol{r}^{\prime}\right)\right] d V^{\prime} .
$$

If the charged body is assumed sufficiently small, $\boldsymbol{B}$ may be taken as constant within the body when evaluating the integrals in Eq. (24) and Eq. (25). Therefore $\boldsymbol{A}(\widetilde{\boldsymbol{r}})$ may be taken as $(1 / 2) \boldsymbol{B}(\boldsymbol{r}) \times \widetilde{\boldsymbol{r}}$ when evaluating these integrals. Assuming a constant charge to mass ratio within the body, gives $\boldsymbol{R}(\boldsymbol{r})$ $=(q / 2 m c) \boldsymbol{B}(\boldsymbol{r}) \times \int \rho \tilde{\boldsymbol{r}} d V^{\prime}=(q / 2 c) \boldsymbol{B}(\boldsymbol{r}) \times \boldsymbol{r}$, where $q$ is the total charge of the body and $m$ is the total mass. Therefore

$$
\boldsymbol{R}(\boldsymbol{r})=\frac{q}{c} \boldsymbol{A}(\boldsymbol{r}) .
$$

To evaluate the integral in Eq. (25) again use $\boldsymbol{A}(\tilde{\boldsymbol{r}})$ $=(1 / 2) \boldsymbol{B}(\boldsymbol{r}) \times \widetilde{\boldsymbol{r}}$ in Eq. (25) and $\widetilde{\boldsymbol{r}}=\boldsymbol{r}+\boldsymbol{r}^{\prime}$. Again assume a constant charge to mass ratio which implies $\int \rho_{q} \boldsymbol{r}^{\prime} d V=0$ and therefore the term in the integral involving $\boldsymbol{r}^{\prime} \times(\boldsymbol{B} \times \boldsymbol{r})$ is zero. Expanding the triple cross product then gives

$$
\boldsymbol{G}(\boldsymbol{r})=\gamma_{c} \boldsymbol{B}(\boldsymbol{r}) \cdot \boldsymbol{I},
$$

where

$$
\gamma_{c}=\frac{q}{2 m c}
$$

is the classical gyromagnetic ratio. Therefore the Lagrangian reduces to

$$
\mathcal{L}=\frac{1}{2} m v^{2}+\frac{q}{c} \boldsymbol{A} \cdot \boldsymbol{v}+\frac{1}{2} \boldsymbol{\omega} \cdot \boldsymbol{I} \cdot \boldsymbol{\omega}+\gamma_{c} \boldsymbol{B} \cdot \boldsymbol{I} \cdot \boldsymbol{\omega},
$$

where $\boldsymbol{A}$ and $\boldsymbol{B}$ are evaluated at the center of mass $\boldsymbol{r}$ of the body. Since the charge to mass ratio within the charged body is assumed to be constant, the magnetic moment is proportional to the mechanical angular momentum $[1,10], \boldsymbol{\mu}$ $=\gamma_{c} \boldsymbol{L}_{m}$. Since $\boldsymbol{L}_{m}=\boldsymbol{I} \cdot \boldsymbol{\omega}$, the last term in Eq. (29) may also be written as $\boldsymbol{\mu} \cdot \boldsymbol{B}$.

As long as the charged body is sufficiently small, the values for $\boldsymbol{R}$ and $\boldsymbol{G}$ given by Eq. (26) and Eq. (27) will at most differ only slightly from the exact values given by Eq. (24) and Eq. (25). Therefore these approximations will cause no qualitative change in the dynamics, but at most only a slight quantitative change. In fact, for the magnetic field given by Eq. (8), and assuming that the center of mass of the charged body is constrained to move on the $z$ axis, that the charged body is spherically symmetric, and that the charge and mass densities are uniform, it may be shown directly from Eq. (25) that $\boldsymbol{G}$ is given exactly by Eq. (27), so that the dynamics is independent of the size of the body. [See the discussion following Eq. (36).]

For $\boldsymbol{v}=0$ and assuming $\boldsymbol{B}$ is independent of position, Eq. (29) reduces to Eq. (41) in Goldstein [1], which gives the Lagrangian for a spinning charged body in a uniform magnetic field. It is interesting that the equations are of the same form, other than the translational terms. The reason for this similarity of appearance, of course, is that $\boldsymbol{B}$ was taken as constant (evaluated at the body's center of mass) when evaluating the integrals. Note, however, that the Lagrangian given by Eq. (29) results in additional dynamics that is qualitatively different, namely the force and translational acceleration resulting from the interaction of the magnetic moment with the magnetic field gradient, as compared with the Lagrangian given by Goldstein. It is the fact that $\boldsymbol{B}$ depends on the center of mass position $\boldsymbol{r}$ that allows for this additional behavior.

Using Eq. (29), the generalized momentum is [9]

$$
p=\frac{\partial \mathcal{L}}{\partial \boldsymbol{v}}=m \boldsymbol{v}+\frac{q}{c} \boldsymbol{A} .
$$

The generalized angular momentum is [9]

$$
\boldsymbol{L}=\frac{\partial \mathcal{L}}{\partial \boldsymbol{\omega}}=\boldsymbol{I} \cdot \boldsymbol{\omega}+\gamma_{c} \boldsymbol{I} \cdot \boldsymbol{B} .
$$

The Hamiltonian is simply the kinetic energy

$$
T=\frac{1}{2} m v^{2}+\frac{1}{2} \boldsymbol{\omega} \cdot \boldsymbol{I} \cdot \boldsymbol{\omega} .
$$

In terms of the generalized momenta, the Hamiltonian is then 


$$
H=\frac{1}{2 m}\left(\boldsymbol{p}-\frac{q}{c} \boldsymbol{A}\right)^{2}+\frac{1}{2} \boldsymbol{L} \cdot \boldsymbol{I}^{-1} \cdot \boldsymbol{L}+\frac{\gamma_{c}^{2}}{2} \boldsymbol{B} \cdot \boldsymbol{I} \cdot \boldsymbol{B}-\gamma_{c} \boldsymbol{L} \cdot \boldsymbol{B} .
$$

The first term is due to the translational motion. The remaining terms are associated with rotational motion. Note that the magnetic moment is not proportional to the generalized angular momentum $\boldsymbol{L}$, but rather to the mechanical angular momentum $\boldsymbol{L}_{m}=\boldsymbol{I} \cdot \boldsymbol{\omega}$. Referring to Eq. (31), the magnetic moment in terms of the generalized angular momentum is

$$
\boldsymbol{\mu}=\gamma_{c} \boldsymbol{L}-\gamma_{c}^{2} \boldsymbol{I} \cdot \boldsymbol{B} .
$$

The expression for the Hamiltonian, Eq. (33), greatly simplifies if the charged body is assumed to be spherically symmetric. Also the behavior is much simpler. If this assumption is not made, then nutation of the spinning body will occur, as pointed out by Goldstein [1] for a uniform magnetic field, which will obscure the behavior of interest. With this assumption, the moment of inertia tensor reduces to the scalar $I$ and the Hamiltonian reduces to

$$
H=\frac{1}{2 m}\left(\boldsymbol{p}-\frac{q}{c} \boldsymbol{A}\right)^{2}+\frac{1}{2 I}\left(\boldsymbol{L}-\gamma_{c} \boldsymbol{I B}\right)^{2}
$$

The first term in this equation corresponds to translational motion and the second term corresponds to rotational motion.

The classical motion of a spinning charged ball (a charged spherical top) in a nonuniform magnetic field is now derived. Taking the magnetic field to be in the positive $z$ direction along the $z$ axis, assuming that the ball is constrained to move on the $z$ axis (causing $\boldsymbol{A} \cdot \boldsymbol{v}=0$ ), and since the ball is spherically symmetric, Eq. (29) becomes

$$
\mathcal{L}=\frac{1}{2} m v^{2}+\frac{1}{2} I \omega^{2}+\gamma_{c} B(z) I \omega_{z}
$$

As noted previously, for the magnetic field given by Eq. (8) and for uniform mass and charge densities within the ball, this expression for $\mathcal{L}$ is exact, valid for any size ball. Referring to Eq. (9), this may be shown by using $A=(1 / 2) r^{\prime}\left[B_{0}\right.$ $\left.+\eta\left(z+z^{\prime}\right)\right] \boldsymbol{u}_{\theta}$ in Eq. (25), where $\boldsymbol{u}_{\theta}$ is the unit vector in the $\theta$ direction and $z$ is the $z$ coordinate of the ball's center of mass. Transforming to spherical coordinates centered at the center of mass for the ball, the integral evaluates identically to $\boldsymbol{G}(z)=\left(q a^{2} / 5 c\right) B(z) \boldsymbol{u}_{z}=\left(q a^{2} / 5 c\right)\left(B_{0}+\eta z\right) \boldsymbol{u}_{z}$, where $a$ is the radius of the charged ball and $\boldsymbol{u}_{z}$ is the unit vector in the $z$ direction. The same expression is obtained from Eq. (27). Therefore the following analysis is valid for any size ball, as long as $\boldsymbol{B}$ is given by Eq. (8) and the charge and mass densities are uniform within the ball.

Since the charged ball is spherically symmetric, the body axes (that is, the axes fixed with the ball, $x^{\prime}, y^{\prime}$, and $z^{\prime}$ ) may be taken in any three mutually perpendicular directions. The Euler angles give the orientation of these axes with respect to the space axes, $x, y$, and $z[10,11]$. The angle $\theta$ gives the amount of tilt of the body axes, that is the angle between $z^{\prime}$ and $z$. The angle $\psi$ gives the angle about the $z^{\prime}$ axis, that is, the angle between the line of nodes and the $x^{\prime}$ axis. The line of nodes is the intersection of the $x-y$ plane and the $x^{\prime}-y^{\prime}$ plane. The angle $\phi$ gives the precession angle, that is the angle between the $x$ axis and the line of nodes. Writing $\omega^{2}$ and $\omega_{z}$ in Eq. (36) in terms of the Euler angles and their derivatives $[1,10,11]$, and using $v=\dot{z}$, the Lagrangian may be written [12]

$$
\begin{aligned}
\mathcal{L}= & \frac{1}{2} m \dot{z}^{2}+\frac{1}{2} I\left(\dot{\phi}^{2}+\dot{\psi}^{2}+\dot{\theta}^{2}+2 \dot{\phi} \dot{\psi} \cos \theta\right)+\gamma_{c} B(z) I(\dot{\phi} \\
& +\dot{\psi} \cos \theta) .
\end{aligned}
$$

Since the Lagrangian is cyclic in $\phi$ and $\psi$ (that is, independent of $\phi$ and $\psi$ ), the momenta conjugate to $\phi$ and $\psi$ are constant:

$$
\begin{gathered}
p_{\phi}=\frac{\partial \mathcal{L}}{\partial \dot{\phi}}=I \dot{\phi}+I \dot{\psi} \cos \theta+\gamma_{c} B(z) I=L_{z}, \\
p_{\psi}=\frac{\partial \mathcal{L}}{\partial \dot{\psi}}=I \dot{\psi}+I \dot{\phi} \cos \theta+\gamma_{c} B(z) I \cos \theta=L .
\end{gathered}
$$

The constant $L_{z}=I \omega_{z}+\gamma_{c} B(z) I$ is simply the $z$ component of the generalized angular momentum. The reason the symbol $L$ is used for the other constant will become apparent later, when it will be shown that $L_{z}=L \cos \theta$, assuming that initial conditions are chosen so that $\theta$ remains constant, that is, there is no nutation.

The equation of motion for the $z$ coordinate is given by Lagrange's equation for $z$,

$$
\frac{d}{d t} \frac{\partial \mathcal{L}}{\partial \dot{z}}-\frac{\partial \mathcal{L}}{\partial z}=0
$$

which gives

$$
m \ddot{z}=\gamma_{c} \eta(z) I(\dot{\phi}+\dot{\psi} \cos \theta)=\gamma_{c} \eta(z)\left[L_{z}-\gamma_{c} B(z) I\right],
$$

where $\eta(z)=\partial B / \partial z$ and where Eq. (38) was used. Assuming a constant magnetic field gradient $\eta$ and taking $B(z)=B_{0}$ $+\eta z$ gives

$$
\ddot{z}=-\omega_{c}^{2}\left(z-z_{0}\right),
$$

where

$$
\omega_{c}=\left|\gamma_{c} \eta\right| \sqrt{\frac{I}{m}}
$$

and

$$
z_{0}=\frac{L_{z}}{\gamma_{c} \eta I}-\frac{B_{0}}{\eta} .
$$

Therefore the spinning charged ball will oscillate in simple harmonic motion along the $z$ axis about the $z$ coordinate given by Eq. (44) and with an angular frequency given by Eq. (43). Again note that a constant magnetic field gradient on the $z$ axis is easily obtained by assuming a magnetic field as given by Eq. (8).

Up to this point, no particular orientation for the body axes have been chosen, nor has the equation of motion for $\theta$ been given. From Lagrange's equation for $\theta$, the equation of motion for $\theta$ is found to be 


$$
\ddot{\theta}+\dot{\phi} \dot{\psi} \sin \theta+\gamma_{c} B(z) \dot{\psi} \sin \theta=0 .
$$

Using Eq. (38) and Eq. (39), the following equation involving only $\theta$ is obtained:

$$
\ddot{\theta}+\frac{\left(L_{z}-L \cos \theta\right)\left(L-L_{z} \cos \theta\right)}{I^{2} \sin ^{3} \theta}=0 .
$$

Choosing the body axes so that $\cos \theta=L_{z} / L$ at $t=t_{0}$ and taking the initial condition $\dot{\theta}=0$ at $t=t_{0}$, Eq. (46) implies that $\theta$ will remain constant (no nutation) even though the charged ball is oscillating along the $z$ axis. Therefore $\ddot{\theta}=0$ and Eq. (45) gives

$$
\dot{\phi}=-\gamma_{c} B(z),
$$

which is the rate of precession for the charged ball. The magnitude of this quantity, $\left|\gamma_{c} B(z)\right|$, is the Larmor frequency. Therefore as the charged ball oscillates along the $z$ axis with an angular frequency given by Eq. (43), it also precesses about the $z$ axis with an angular frequency given by the magnitude of Eq. (47). Note that the Larmor frequency is a function of $z$ and therefore is a function of time.

Using Eq. (47) in Eq. (38) and in Eq. (39), gives for $\dot{\psi}$ and for the relationship between $L_{z}$ and $L$

$$
\dot{\psi}=\frac{L}{I}
$$

and

$$
L_{z}=L \cos \theta .
$$

Therefore as the charged ball oscillates along the $z$ axis, it is only the precession rate $\dot{\phi}$ which changes. The rotation rate about the $z^{\prime}$ axis, $\dot{\psi}$, remains constant.

The kinetic energy in terms of the velocity in the $z$ direction and in terms of the Euler angles and their derivatives is just the kinetic energy part of Eq. (37). Taking $\dot{\theta}=0$ gives

$$
T=\frac{1}{2} m v^{2}+\frac{1}{2} I\left(\dot{\psi}^{2}+\dot{\phi}^{2}+2 \dot{\phi} \dot{\psi} \cos \theta\right) .
$$

Since $\dot{\psi}$ is constant, the only variable that changes in the rotational part of $T$ is $\dot{\phi}$. Therefore as the charged ball accelerates along the $z$ axis, any change in $v$ is associated with a change in $\dot{\phi}$, such that $T$ remains constant. So any increase in translational kinetic energy results from a corresponding decrease in rotational kinetic energy associated with a change in the precession rate for the charged ball. Note that the direction of precession may be opposite to the direction of rotation about the $z^{\prime}$ body axis (i.e., the figure axis). Therefore an increase in the magnitude of the precession rate can correspond to a decrease in rotational kinetic energy. Also it is interesting to note that, for a given rotation rate $\dot{\psi}$ about the figure axis, a change from $\theta$ to $\pi-\theta$ (a "spin flip"), corresponds to a change in rotational kinetic energy, even for a uniform magnetic field. This is so since the directions of precession are the same for both states, while the projections of the rotation vectors about the figure axes onto the $z$ axis are opposite. This may also be seen from Eq. (50).
Using Eq. (47), Eq. (48), and Eq. (49) in Eq. (50) gives

$$
T=\frac{1}{2} m v^{2}+\frac{L^{2}}{2 I}+\frac{1}{2} I \gamma_{c}^{2} B(z)^{2}-\gamma_{c} B(z) L_{z} .
$$

This equation is precisely that given by the Hamiltonian of Eq. (35), assuming the magnetic field to be in the positive $z$ direction along the $z$ axis and assuming that the particle is constrained to move on the $z$ axis.

The $z$ component of the magnetic moment in terms of the Euler angles and their derivatives is

$$
\mu_{z}=\gamma_{c} I \omega_{z}=\gamma_{c} I(\dot{\psi} \cos \theta+\dot{\phi}) .
$$

Thus there are two contributions to the magnetic moment: rotation about the $z^{\prime}$ body axis and precessional rotation about the $z$ axis. Using Eq. (47), Eq. (48), and Eq. (49) in Eq. (52) gives

$$
\mu_{z}=\gamma_{c} L_{z}-\gamma_{c}^{2} I B(z),
$$

which agrees with Eq. (34). Note that $z_{0}$ in Eq. (44), the value about which the ball oscillates, is also given by the value of $z$ which minimizes $(1 / 2) I \gamma_{c}^{2} B(z)^{2}-\gamma_{c} B(z) L_{z}$ in Eq. (51) and by the value of $z$ for which the magnetic moment, Eq. (53), is zero.

\section{AN ATOM IN A NONUNIFORM MAGNETIC FIELD}

The nonrelativistic quantum Hamiltonian for a single atom in a nonuniform magnetic field is now derived. Landau and Lifshitz [3] have derived the Hamiltonian for an atom in a uniform magnetic field. Their derivation is now generalized to include a nonuniform magnetic field and a nonzero translational velocity. Taking the center of mass to be at the nucleus, the classical Lagrangian is

$$
\begin{aligned}
\mathcal{L}= & \frac{1}{2} m_{n} V^{2}+\sum_{i} \frac{1}{2} m_{e}\left(\boldsymbol{V}+\boldsymbol{v}_{i}\right)^{2} \\
& +\frac{Z e}{c} \boldsymbol{A} \cdot \boldsymbol{V}-\sum_{i} \frac{e}{c} \boldsymbol{A}_{i} \cdot\left(\boldsymbol{V}+\boldsymbol{v}_{i}\right)-U,
\end{aligned}
$$

where $m_{n}$ is the nuclear mass, $m_{e}$ is the electron mass, $e$ is the magnitude of the electron charge, $Z$ is the atomic number, $\boldsymbol{V}$ is the center of mass velocity of the atom, $\boldsymbol{v}_{i}$ is the velocity of the $i$ th electron relative to the center of mass, $A=\boldsymbol{A}(\boldsymbol{R})$ is the vector potential at the position $\boldsymbol{R}$ of the nucleus, $\boldsymbol{A}_{i}$ $=\boldsymbol{A}\left(\widetilde{\boldsymbol{r}}_{i}\right)$ is the vector potential at the position $\widetilde{\boldsymbol{r}}_{i}=\boldsymbol{R}+\boldsymbol{r}_{i}$ of the $i$ th electron in a fixed reference frame, $\boldsymbol{r}_{i}$ is the position of the $i$ th electron relative to the center of mass, $U=U\left(\boldsymbol{r}_{i}\right)$ is the potential energy of interaction of the electrons with the nucleus and with each other, and the sums are taken over all $Z$ electrons.

As in the derivation leading to Eq. (29) for the classical dipole, it is assumed that the magnetic field changes little over the volume of the atom, so that $\boldsymbol{B}_{i}=\boldsymbol{B}\left(\widetilde{\boldsymbol{r}}_{i}\right)$ may be taken as constant within the atom, that is $\boldsymbol{B}_{i}=\boldsymbol{B}(\boldsymbol{R})$, and thus $\boldsymbol{A}_{i}$ $=(1 / 2) \boldsymbol{B}(\boldsymbol{R}) \times \widetilde{\boldsymbol{r}}_{i}$ when evaluating the sums. Expanding and simplifying, Eq. (54) reduces to 


$$
\mathcal{L}=\frac{1}{2} M V^{2}+\sum_{i} \frac{1}{2} m_{e} v_{i}^{2}-\sum_{i} \frac{e}{2 c}\left(\boldsymbol{B} \times \boldsymbol{r}_{i}\right) \cdot \boldsymbol{v}_{i}-U,
$$

where $M$ is the total mass of the atom and $\boldsymbol{B}=\boldsymbol{B}(\boldsymbol{R})$. Using $\boldsymbol{P}=\partial \mathcal{L} / \partial \mathbf{V}$ and $\boldsymbol{p}_{i}=\partial \mathcal{L} / \partial \mathbf{v}_{\mathbf{i}}$ for the momenta conjugate to $\boldsymbol{R}$ and $\boldsymbol{r}_{i}$, respectively, and adding a term involving the interaction of the electron spins with the magnetic field, the quantum Hamiltonian is

$$
H=\frac{P^{2}}{2 M}+\frac{1}{2 m} \sum_{i}\left(\boldsymbol{p}_{i}+\frac{e}{2 c} \boldsymbol{B} \times \boldsymbol{r}_{i}\right)^{2}+U+\frac{e}{m c} \sum_{i} \boldsymbol{B} \cdot \boldsymbol{S}_{i},
$$

where $\boldsymbol{P}$ is the momentum operator for the atom, $\boldsymbol{p}_{i}$ is the momentum operator for the $i$ th electron, and $S_{i}$ is the spin angular momentum operator for the $i$ th electron. The first term is related to the translational kinetic energy of the atom as a whole, the second and third terms are related to the internal energy of the atom, that is, the kinetic energy plus potential energy in the atom's rest frame, and the last term is related to the interaction energy of the spin with the magnetic field. After some manipulation and noting that the orbital angular momentum of an electron is $\boldsymbol{r}_{i} \times \boldsymbol{p}_{i}, H$ may be written as

$$
H=H_{t}+H_{e},
$$

where

$$
H_{t}=\frac{P^{2}}{2 M}
$$

and

$$
H_{e}=H_{0}+\frac{e}{2 m c}(\boldsymbol{L}+2 \boldsymbol{S}) \cdot \boldsymbol{B}(\boldsymbol{R})+\frac{e^{2}}{8 m c^{2}} \sum_{i}\left[\boldsymbol{B}(\boldsymbol{R}) \times \boldsymbol{r}_{i}\right]^{2},
$$

where $H_{t}$ is related to the translational kinetic energy of the atom as a whole, $H_{e}$ is related to the electronic energy relative to the center of mass (including the spin-field interaction energy), $H_{0}$ is the Hamiltonian in the atom's rest frame in the absence of the magnetic field, $\boldsymbol{B}(\boldsymbol{R})$ is the magnetic field at the position of the nucleus $\boldsymbol{R}, \boldsymbol{L}$ is the total angular momentum operator due to orbital motion of all the electrons relative to the nucleus, $S$ is the total spin angular momentum operator due to the spin of all the electrons, and $\boldsymbol{r}_{i}$ is the position of the $i$ th electron relative to the nucleus.

Although $\boldsymbol{L}$ and $\boldsymbol{S}$ appear to be on equal footing in Eq. (59), note that $(e / 2 m c) \boldsymbol{L} \cdot \boldsymbol{B}$ is simply part of the internal energy of the atom, whereas $(e / m c) \boldsymbol{S} \cdot \boldsymbol{B}$ is the spin-field interaction energy. Whether or not the magnetic field does work when there is a change in the spin-field energy depends on whether the electron has rotational kinetic energy associated with its spin. Since nonrelativistic theory was the starting point in deriving the Hamiltonian, Eq. (59), the spin-field energy was simply added $[3,6]$. If the starting point is relativistic quantum theory, then the spin-field interaction energy will arise naturally $[5,6]$, which will be discussed in the last section of the present paper.
Without the translational term and for uniform $\boldsymbol{B}$, Eqs. (57)-(59) reduce to Eq. (112.1) in Landau and Lifshitz [3], which is the Hamiltonian for an atom at rest in a uniform magnetic field. Therefore the Hamiltonian given by Eqs. (57)-(59) differs from the Hamiltonian of Landau and Lifshitz by only the translational term $P^{2} / 2 M$. Similar to the case of the classical dipole, the reason for this is that the magnetic field was taken as constant within the atom when evaluating the sums in Eq. (54). Although the center of mass kinetic energy may appear to be a trivial addition to the Hamiltonian of Landau and Lifshitz, note that in combination with allowing the magnetic field to depend on the position of the center of mass of the atom, qualitatively different behavior results, namely the force and translational acceleration of the atom resulting from the interaction of the magnetic moment with the magnetic field gradient.

Because of the interaction of the magnetic moment with the magnetic field gradient, the atom will accelerate. The rate of change of the expectation value of the translational momentum of the atom, $\langle\boldsymbol{P}\rangle$, is found by generalizing the derivation of Ehrenfest's theorem [4]. Noting that the expectation value of the Laplacian associated with the motion of the atom as a whole and the Laplacians associated with the motion of the electrons (present in $H_{0}$ ) may be converted to surface integrals which vanish at large distances, and that the potential present in $H_{0}$ is independent of position of the atom, the rate of change of the expectation value of the translational momentum is

$$
\begin{aligned}
\frac{d\langle\boldsymbol{P}\rangle}{d t}= & -\frac{e}{2 m c}\langle\nabla[(\boldsymbol{L}+2 \boldsymbol{S}) \cdot \boldsymbol{B}(\boldsymbol{R})]\rangle \\
& -\frac{e^{2}}{8 m c^{2}} \sum_{i}\left\langle\nabla\left[\left(\boldsymbol{B}(\boldsymbol{R}) \times \boldsymbol{r}_{i}\right)^{2}\right]\right\rangle .
\end{aligned}
$$

The gradient in Eq. (60) is the gradient related to the position of the atom as a whole, that is, $\boldsymbol{\nabla}=\partial / \partial \boldsymbol{R}$.

Any change in the expectation value of the translational momentum will be associated with a change in the translational kinetic energy of the atom. In general, any operator which commutes with the Hamiltonian is a constant of the motion [4]. Since $H$ commutes with itself, the energy $E$ $=\langle H\rangle$ is a constant of the motion, where $H$ is given by Eqs. (57)-(59). If $S=0$, then $\left\langle H_{e}\right\rangle$ is simply the internal energy of the atom. Since $E=\langle H\rangle=\left\langle H_{t}\right\rangle+\left\langle H_{e}\right\rangle$ is a constant of the motion, any increase in the translational kinetic energy $\left\langle H_{t}\right\rangle$ corresponds to an equal decrease in the internal energy $\left\langle H_{e}\right\rangle$ (with $S=0$ ), and no work is done by the magnetic field. For example, take the magnetic field to be in the positive $z$ direction along the $z$ axis, assume a positive magnetic field gradient on the $z$ axis, and assume that the atom's center of mass is constrained to move on the $z$ axis. Referring to Eq. (60), if $L_{z}>0$ and $S_{z}=0$ [the last term in Eq. (60) being assumed small], the atom will accelerate in the negative $z$ direction and, as $\left\langle H_{t}\right\rangle$ increases, $\left\langle H_{e}\right\rangle$ decreases by the same amount. Similarly, if $L_{z}<0$ and $S_{z}=0$, the atom will accelerate in the positive $z$ direction and again, as $\left\langle H_{t}\right\rangle$ increases, $\left\langle H_{e}\right\rangle$ decreases by the same amount. Thus the source of the translational kinetic energy is the internal energy of the atom, and the magnetic field does no work. Note that if both $S$ and 
$\boldsymbol{L}$ are zero, an atom will still accelerate due to the nonlinear term. This is the term responsible for diamagnetism [3].

If $\boldsymbol{S} \cdot \boldsymbol{B}$ were not related to the rotational kinetic energy of the electron, but rather to a "potential energy" due to the interaction of the electron's spin with the magnetic field, then if $\boldsymbol{S}$ were nonzero, the magnetic field would be doing work if there were any change in $\boldsymbol{S} \cdot \boldsymbol{B}$. For example, referring to Eq. (60), if a silver atom $(\boldsymbol{L}=0, \boldsymbol{S} \neq 0)$ is constrained to move on the $z$ axis for the magnetic field of the last paragraph and if $S_{z}<0$ [the last term in Eq. (60) being assumed small], the atom will accelerate in the positive $z$ direction. As $\left\langle H_{t}\right\rangle$ increases, there will be a corresponding decrease in $\left\langle H_{e}\right\rangle$ by the same amount. Now, however, the magnetic field would be doing work, since $\boldsymbol{S} \cdot \boldsymbol{B}$ would be changing and the electron spin would have no internal energy (rotational kinetic energy) associated with it, but only a "potential energy" associated with the interaction between the electron spin and the magnetic field. If both $S \neq 0$ and $\boldsymbol{L} \neq 0$, then, as previously pointed out in the introduction, the magnetic field would not be doing work on the orbital contribution to the magnetic moment, while at the same time doing work on the spin contribution.

An interesting question is whether an atom can be bound in a constant magnetic field gradient, similar to what occurred in the classical problems of the oscillating rotating charged ring and the oscillating spinning charged ball. This question may be investigated by using the BornOppenheimer approximation [4] to simplify the Schrödinger equation $H \psi=E \psi$, where $H$ is given by Eqs. (57)-(59). This approximation may be used since the mass of the atom is much larger than the mass of the electrons and therefore at any given instant, the energy of the electrons is determined by the Schrödinger equation for the electrons in the rest frame of the atom, this energy changing only slowly as the position of the atom changes. Assuming a solution of the form

$$
\psi\left(\boldsymbol{r}_{i}, \boldsymbol{R}\right)=\varphi\left(\boldsymbol{r}_{i} ; \boldsymbol{R}\right) \chi(\boldsymbol{R}),
$$

$\varphi\left(\boldsymbol{r}_{i} ; \boldsymbol{R}\right)$ is the wave function corresponding to the motion of the electrons in the rest frame of the atom and $\chi(\boldsymbol{R})$ is the wave function corresponding to the motion of the atom as a whole. Here $\boldsymbol{R}$ is the position of the center of mass of the atom and $\boldsymbol{r}_{i}$ corresponds to the positions of the electrons relative to the center of mass. The parameter $\boldsymbol{R}$ in $\varphi\left(\boldsymbol{r}_{i} ; \boldsymbol{R}\right)$ is a slowly varying parameter. Substituting the solution Eq. (61) into Eqs. (57)-(59) gives approximately

$$
H_{e} \varphi\left(\boldsymbol{r}_{i} ; \boldsymbol{R}\right)=E_{e}(\boldsymbol{R}) \varphi\left(\boldsymbol{r}_{i} ; \boldsymbol{R}\right),
$$

where $H_{e}$ is given by Eq. (59), $E_{e}(\boldsymbol{R})$ is the energy of the electrons in the rest frame of the atom, and $\boldsymbol{R}$ is assumed fixed; and

$$
\left[H_{t}+E_{e}(\boldsymbol{R})\right] \chi(\boldsymbol{R})=E \chi(\boldsymbol{R}) .
$$

Assuming the atom is constrained to move on the $z$ axis, $\boldsymbol{R}$ is replaced by $Z$ in Eqs. (59), (62), and (63), where $Z$ is the position of the center of mass of the atom on the $z$ axis. If $E_{e}(Z)$ in Eq. (63) has an absolute minimum, then the atom will be bounded on the $z$ axis about the $Z$ value where $E_{e}(Z)$ is minimum. If the gradient on the $z$ axis is constant, that is
$B(Z)=B_{0}+\eta Z$, then $E_{e}(Z)$ will have a minimum if $E_{e}(B)$ has a minimum, where $B$ is the magnitude of $\boldsymbol{B}$. If $(\boldsymbol{L}+2 \boldsymbol{S}) \cdot \boldsymbol{B}$ $<0$ in Eq. (59) (that is, $\boldsymbol{\mu}_{a} \cdot \boldsymbol{B}>0$, where $\boldsymbol{\mu}_{a}$ is the atom's intrinsic magnetic moment or the magnetic moment in the absence of $\boldsymbol{B})$, then as $B$ is increased from zero, $E_{e}(B)$ will decrease. The question then is whether the quadratic term in $B$ will cause $E_{e}(B)$ to increase for sufficiently large $B$.

It turns out that for the ground state of a hydrogen atom, $E_{e}(B)$ is a monotonically decreasing function of $B$ as $B$ is increased from zero up to $4.7 \times 10^{12} \mathrm{G}$ [13]. Therefore a hydrogen atom cannot be bound along the $z$ axis by a constant gradient magnetic field along the $z$ axis. For lithium, there is a minimum for the $1 s^{2} 2 p_{-1}$ state at about $B=3.7$ $\times 10^{9} \mathrm{G}[14,15]$. However, other states are monotonically decreasing. In particular, the $1 s 2 p_{-1} 3 d_{-2}$ state is monotonically decreasing and becomes less than the minimum of the $1 s^{2} 2 p_{-1}$ state at about $B=5.3 \times 10^{9} \mathrm{G}[15]$. Therefore at best there is a local minimum, and the lithium atom could at best be bound only temporally by a constant gradient magnetic field along the $z$ axis. Similar considerations would hold for other atoms such as carbon [14].

\section{WORK AND QUANTUM SPIN}

The Dirac equation for a relativistic electron in a static magnetic field $\boldsymbol{B}=\boldsymbol{\nabla} \times \boldsymbol{A}$ is [5]

$$
i \hbar \frac{\partial \psi}{\partial t}=\left[c \boldsymbol{\alpha} \cdot\left(\boldsymbol{p}+\frac{e}{c} \boldsymbol{A}\right)+\beta m c^{2}\right] \psi=H \psi,
$$

where $e$ is the magnitude of the electron charge and where

$$
\alpha^{i}=\left(\begin{array}{cc}
0 & \sigma^{i} \\
\sigma^{i} & 0
\end{array}\right) \text { and } \beta=\left(\begin{array}{cc}
I & 0 \\
0 & -I
\end{array}\right),
$$

where $\sigma^{i}$ are the Pauli spin matrices

$$
\sigma^{1}=\left(\begin{array}{ll}
0 & 1 \\
1 & 0
\end{array}\right), \quad \sigma^{2}=\left(\begin{array}{cc}
0 & -i \\
i & 0
\end{array}\right), \quad \sigma^{3}=\left(\begin{array}{cc}
1 & 0 \\
0 & -1
\end{array}\right),
$$

and $I$ is the two-dimensional identity matrix. Equation (64) has two positive-energy solutions, corresponding to the electron, and two negative-energy solutions, corresponding to the positron.

As noted in the Introduction, a rotational kinetic energy for the electron is consistent with the Dirac equation. Since $\boldsymbol{p}$ in Eq. (64) is the momentum operator related to the translational motion of the electron, the question is in what term does the rotational kinetic energy appear? Noting that an electron at rest has spin, any energy associated with this spin would be contained in the electron's rest energy. Therefore the quantity $m c^{2}$ should be identified as the rest energy in the absence of a magnetic field. In the presence of a magnetic field, the electron would precess, thereby slightly changing the rotational kinetic energy. If the spin angular momentum is in the same direction as the precession, the rotational kinetic energy would increase. If the spin angular momentum is in the opposite direction as the precession, the rotational kinetic energy would decrease. An important point is that no modification to the Dirac equation is required. 
This behavior is similar to that of the classical magnetic dipole, where precession of the dipole similarly affects the rotational kinetic energy. [See the discussion following Eq. (50).] However, it is important to note that a model of the electron as a rigid rotator is not implied [16]. Here it is only assumed, along the lines of Merzbacher [4], that the electron's spin angular momentum and magnetic moment are the result of "some (admittedly unanalyzable or, at least, unanalyzed) internal circulating currents of charged matter." Since these circulating currents would also precess due to the interaction of the spin angular momentum with the magnetic field, any rotational kinetic energy associated with these circulating currents would be modified slightly as a result of the precession.

This may be seen more clearly by finding the equation satisfied by the positive-energy solutions of the Dirac equation in the nonrelativistic limit. Following Schwabl [5], write

$$
\psi=\left(\begin{array}{c}
\tilde{\varphi} \\
\tilde{\chi}
\end{array}\right)=e^{-i\left(m c^{2} / \hbar\right) t}\left(\begin{array}{c}
\varphi \\
\chi
\end{array}\right),
$$

where $\varphi$ and $\chi$ are slowly varying with time. In the nonrelativistic limit and after considerable manipulation [5], the equation for $\varphi$ reduces to

$$
i \hbar \frac{\partial \varphi}{\partial t}=\left[\frac{1}{2 m}\left(\boldsymbol{p}+\frac{e}{c} \boldsymbol{A}\right)^{2}+\frac{e \hbar}{2 m c} \boldsymbol{\sigma} \cdot \boldsymbol{B}\right] \varphi,
$$

which is just Pauli's equation (Schrödinger's equation with spin). The spin angular momentum is $S=(\hbar / 2) \boldsymbol{\sigma}$ and the spin magnetic moment is $\boldsymbol{\mu}_{s}=-(e \hbar / 2 m c) \boldsymbol{\sigma}$, giving the correct value for the electron's magnetic moment (neglecting radiative effects).

To see how the rest energy enters the nonrelativistic equation, return to the variable $\widetilde{\varphi}$ by using $\widetilde{\varphi}=e^{-i\left(m c^{2} / \hbar\right) t} \varphi$, which gives

$$
i \hbar \frac{\partial \widetilde{\varphi}}{\partial t}=\left[\frac{1}{2 m}\left(\boldsymbol{p}+\frac{e}{c} \boldsymbol{A}\right)^{2}+m c^{2}+\frac{e \hbar}{2 m c} \boldsymbol{\sigma} \cdot \boldsymbol{B}\right] \widetilde{\varphi} .
$$

Again, the term $m c^{2}$ is the rest energy of the electron in the absence of a magnetic field and would contain any rotational kinetic energy associated with the spin. In the presence of a magnetic field the electron's spin angular momentum will precess about the magnetic field vector [3,4], the direction of precession being the same for both spin-up and spin-down states. If the spin angular momentum is in the same direction as the precession, the rotational kinetic energy would differ by $e \hbar B / 2 m c$ from the rotational kinetic energy in the ab- sence of the magnetic field. If the spin angular momentum is in the opposite direction as the precession, the rotational kinetic energy would differ by $-e \hbar B / 2 m c$ from the rotational kinetic energy in the absence of the magnetic field.

So far in the present section, the equations describe a single electron in a magnetic field. If there are multiple electrons in an atom, then the Dirac equation needs to be modified by adding electron-electron interaction terms [6]. Since the nonrelativistic limit is the primary interest in the present paper, it is sufficient to add a Coulomb electron-electron interaction term to the Dirac equation for $N$ electrons. In the nonrelativistic limit, the equation $i \hbar \partial \varphi / \partial t=H_{e} \varphi$ results, where $H_{e}$ is given by Eq. (59). In the variable $\widetilde{\varphi}, H_{e}$ in Eq. (59) will have the additional term $N m c^{2}$.

As an application of these concepts, consider an electron making a transition from a spin-up to a spin-down state in a magnetic field, thereby emitting a photon. The usual explanation is that there is a change in the "potential energy" by an amount $-2\left|\boldsymbol{\mu}_{s} \cdot \boldsymbol{B}\right|=-e \hbar B / m c$, which implies that the magnetic field did work on the electron's magnetic moment. However, if the electron has rotational kinetic energy, then the emission of the photon can be explained as follows. As noted above, since the direction of precession is the same for both spin-up and spin-down states, the rotational kinetic energy of spin-up and spin-down states would be different. An electron making a transition from spin up to spin down would then correspond to a change of $-e \hbar B / m c$ in the rotational kinetic energy of the electron, and therefore the magnetic field would do no work.

Similarly, the increase in the translational kinetic energy of a silver atom with a spin-up unpaired electron, as it accelerates into a region with a weaker magnetic field in a SternGerlach experiment, can also be explained, without requiring the magnetic field to do work, by noting that the precession frequency of the electron decreases, thereby decreasing the rotational kinetic energy of the electron. For a spin-down electron, the precession frequency is opposite to the spin, so the increased translational kinetic energy and increased electron precession frequency as the silver atom accelerates into a stronger magnetic field region also corresponds to a decrease in rotational kinetic energy.

\section{ACKNOWLEDGMENTS}

I thank Jearl Walker, who initiated this investigation by asking me the question: Can a magnetic field do work on a dipole? I also thank Jearl Walker, James Lock, and Steven Marx for stimulating discussions, as well as for helpful comments on the manuscript.
[1] H. Goldstein, Am. J. Phys. 19, 100 (1951).

[2] C. A. Coombes, Am. J. Phys. 47, 915 (1979).

[3] L. D. Landau and E. M. Lifshitz, Quantum Mechanics (Pergamon Press, Oxford, 1965).

[4] E. Merzbacher, Quantum Mechanics (Wiley, New York, 1970).

[5] F. Schwabl, Advanced Quantum Mechanics, translated by R.
Hilton and A. Lahee (Springer-Verlag, Berlin, Heidelberg, 2004).

[6] P. Strange, Relativistic Quantum Mechanics (Cambridge University Press, Cambridge, UK, 1998).

[7] J. D. Jackson, Classical Electrodynamics (John Wiley and Sons, New York, 1962). 
[8] Gaussian units are used in the present paper.

[9] The quantity $\partial \mathcal{L} / \partial \boldsymbol{v}$ is referred to as the generalized momentum, since it is equal to the mechanical momentum plus an additional term involving $\boldsymbol{A}[10]$. Similarly, $\partial \mathcal{L} / \partial \boldsymbol{\omega}$ is referred to as the generalized angular momentum, since it is equal to the mechanical angular momentum plus an additional term involving $\boldsymbol{B}$. The terms conjugate or cononical momenta may also be used for these momenta [10].

[10] H. Goldstein, Classical Mechanics (Addison-Wesley, Reading, MA, 1950)

[11] L. D. Landau and E. M. Lifshitz, Mechanics (Pergamon Press, Oxford, 1976).

[12] If comparing Eq. (37) with Eq. (16) in Goldstein [1], note that there is a typographical error in Eq. (16) of Goldstein, namely $\dot{\phi}$ should read $\dot{\psi}$ in the last term of this equation. There is a similar error in Eq. (4).

[13] J. Xi, L. Wu, X. He, and B. Li, Phys. Rev. A 46, 5806 (1992).

[14] M. D. Jones, G. Ortiz, and D. M. Ceperley, Phys. Rev. A 54, 219 (1996).

[15] M. V. Ivanov and P. Schmelcher, Phys. Rev. A 57, 3793 (1998).

[16] Problems with a model of the electron as a rigid body include (i) speeds which greatly exceed the speed of light for a point on the edge of the electron, when the Coulomb energy is equated to the rest energy; and (ii) integral spin instead of half integral spin. 